Review

\title{
Immunobiology of the blood-brain barrier
}

\author{
Donald W Miller ${ }^{* 1}$ \\ ${ }^{1}$ Department of Pharmaceutical Sciences, College of Pharmacy, University of Nebraska Medical Center, 986025 \\ Nebraska Medical Center, Omaha, Nebraska, NE 68198-6025, USA
}

\begin{abstract}
The brain microvessel endothelial cells (BMVEC) that form the blood-brain barrier are uniquely positioned to influence immune responses within the central nervous system. As the biological interface separating the blood from the brain extracellular fluid, BMVEC regulate the entry of leukocytes into the brain. In addition, through the release of various soluble factors that affect immune responses, BMVEC may modulate immune responses in the brain. This review addresses the interplay between the immune system and the blood-brain barrier as it relates to the regulation of CNS defense and immunity.
\end{abstract}

Keywords: leukocyte migration; endothelial cells; modulation

\section{Introduction}

The brain has often been considered an immunologically privileged organ. This was based on early studies that found few antigen-presenting cells in the central nervous system. In addition, there was a perceived lack of a lymphatic system within the brain to carry immunogenic material in the central nervous system to lymph nodes where a humoral immune response could be initiated. And finally, the presence of the blood-brain barrier (BBB) was thought to prevent the entry of immune cells from the peripheral circulation into the brain.

However, there is increasing evidence to suggest that the brain is under immunological surveillance (see reviews by Cserr and Knopf, 1992; Hickey, 1999). First, the glial cells within the brain can secrete cytokines and chemokines as well as participate in antigen presentation (Ransohoff and Tani, 1998; O'Keefe et al, 1999). Furthermore, the failure of most long term central nervous system xenografts are the result of immune responses within the brain (Czech et al, 1997). From a disease perspective, multiple sclerosis (MS) and experimental allergic encephalomyelitis (EAE) clearly demonstrate the devastation brought about by cellular and humoral immune responses within the central nervous system.

The BBB was once considered to be a physical, static barrier to the passage of solutes and macromolecules from the bloodstream to the brain

${ }^{*}$ Correspondence: DW Miller

Received 20 July 1999; revised 8 September 1999; accepted 10 September 1999 extracellular fluid. However, recent studies indicate the BMVEC of the BBB form a dynamic interface between the blood and the brain that can be influenced by drug regimens (Bartus et al, 1996; Greig, 1989) and endogenous factors such as bradykinin (Hurst and Clark, 1998) bile salts (Greenwood et al, 1991) and cytokines (Mark and Miller, 1999). Changes in BBB function are observed in several neurological disorders associated with immune responses (i.e. MS, meningitis, HIV-1associated dementia) (Sharief et al, 1993; Spellerberg and Tuomanen, 1994; Epstein, 1998; Power and Johnson, 1995). The alterations in BBB function observed in the above neurological disorders result in a loss of integrity and enhanced permeability of the BMVEC. The extent to which BBB permeability is increased in neurological disorders such as MS and meningitis is positively correlated with the severity of the disease (Sharief et al, 1993; Spellerberg and Tuomanen, 1994). Therefore, understanding the interactions of the immune system with the $\mathrm{BBB}$ during both health and disease may provide insight into the treatment of various neurological diseases.

This review examines the interplay between the immune response and the BBB. Special emphasis will be given to examining leukocyte adherence to and penetration through the BBB. Although there are several types of leukocytes, the present review will focus on the neutrophils, monocytes and lymphocytes ( $\mathrm{T}$ and $\mathrm{B}$ cells) and their movement across the BBB. In addition, the review will consider how the BBB can influence immune 
responses within the central nervous system by controlling the entry of immunomodulatory agents into the brain. In this regard, the BBB will be treated as both a target site for the immune system, and a conduit for activation of immune responses within the central nervous system.

\section{Components of the BBB}

The schematic in Figure 1 compares the capillaries in the peripheral vasculature to those that form the BBB. In contrast to many of the endothelial cells found in the peripheral microvasculature, the BMVEC form a continuous layer of cells and extracellular matrix (Audus et al, 1992; Broadwell, 1989). There are also no detectable fenestrations and reduced levels of pinocytic activity in the BMVEC that form the BBB compared to endothelial cells in the peripheral microvasculature (Audus et al, 1992; Broadwell, 1989). In addition, the brain microvessel endothelial cells also form tight extracellular junctions, similar (but not identical) to those found in epithelial cells (Schneeberger and Lynch, 1984; Brightman, 1989). Together, these morphological features act to limit the passage of solutes, macromolecules and cells across the BBB.

Astrocytes are an additional cellular component of the BBB. The astrocytes are a type of glial cell that extend foot-like projections around the outside of the capillaries (see Figure 1). While the astrocytes themselves do not form the barrier, they do have an important role in the development and maintenance of the BBB (Goldstein, 1988). The importance of the astrocytes to the establishment of the BBB comes from the seminal work of Stewart and Wiley (1981). In these studies, the embryonic, avascular quail brain was implanted into the abdominal cavity of a chick embryo. Although the resulting microvasculature in the implanted quail brain originated from the systemic abdominal vasculature of the chick, distinct functional, morphological and biochemical features emerged characteristic of the BBB. These studies indicated that endothelial cells placed in the microenvironment of the brain, develop the characteristics of the brain microvessels that form the BBB (Stewart and Wiley, 1981).

Direct evidence also exists that confirms the importance of the astrocytes in influencing the barrier properties of brain endothelial cells. These are through the use of astrocyte co-culture and astrocyte conditioned media in cell culture models of the BBB (Rubin et al, 1991; Raub et al, 1992; Hurwitz et al, 1993; Rist et al, 1997; Hayashi et al, 1997). In these models, astrocytes were either grown on the underside of a semi-permeable membrane with the endothelial cells cultured on the opposite side of the membrane, or media was collected from astrocytes and then used in the media provided to the endothelial cells. The use of astrocyte co-culture and conditioned media have been reported to reduce the permeability of the BMVEC (Rubin et al, 1991; Raub et al, 1992; Rist et al, 1997). Even endothelial cells from the peripheral microvasculature (i.e. umbilical vein endothelial cells) displayed reduced permeability and BBB-specific characteristics following co-culture with astrocytes (Hurwitz et al, 1993; Hayashi et al, 1997). Identification of the astrocyte-released

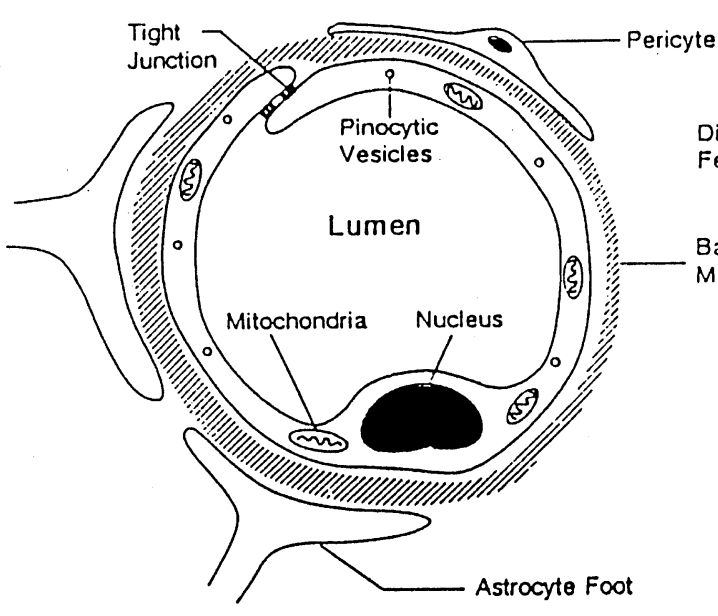

Brain Capillary

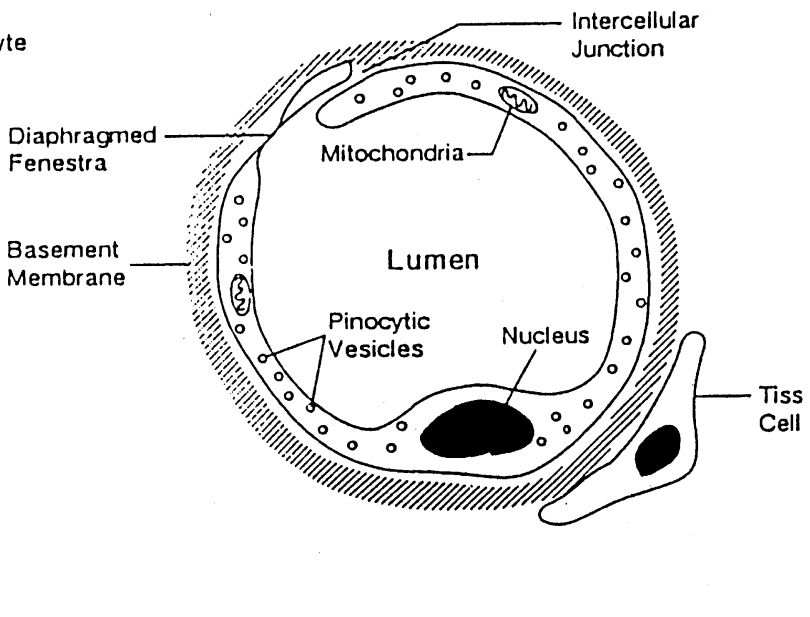

Peripheral Capillary

Figure 1 Cross-sectional diagram comparing brain capillary and peripheral capillary. Significant differences include the presence of tight junctions, reduced pinocytic vesicles, increased mitochondria organelles and the close association to astrocytes in the brain capillary. 
factors involved in controlling the permeability properties of the brain endothelial cells, as well as the cellular mechanisms responsible for such permeability changes, remain unknown. However, it is clear that the astrocytes can influence the permeability characteristics of the BMVEC that form the BBB.

In addition to the effects of astrocytes on BMVEC permeability, it has also been suggested that the astrocytes may influence the expression of adhesion molecules in the brain microvessel endothelial cells that form the BBB (Engelhardt et al, 1997). Studies by Joseph and colleagues (1997), demonstrated an increased adhesion of $\mathrm{T}$ cells to both brain and peripheral endothelia cells following exposure to astrocyte conditioned media. In this regard, the coculturing of human brain microvessel endothelial cells with human astrocytes may offer a more representative model for examining immune responses in the BBB.

Although they occur less frequently than the astrocytes, pericytes and perivascular cells can also be found scattered along the length of the brain capillary endothelial cells. The pericytes are smooth muscle cells that are in close proximity to the BMVEC (see Figure 1). Although the function of the pericytes in the BBB are still being debated, these cells produce and secrete several immunomodulating agents that can influence immune responses in the CNS (Fabry et al, 1993). The pericytes may also provide a means for contraction of the brain capillaries. Perivascular cells are distinct from the smooth muscle derived pericytes. The perivascular cells are also believed to have an immune function in the CNS (Angelov et al, 1998). Studies suggest the perivascular cells, located near the basal lamina of the BMVEC, can act as macrophages capable of phagocytosis and antigen presentation (Angelov et al, 1998). Given the proposed function of these cells and their proximity to the brain capillaries, the cerebral perivascular cells may provide a first defense to infiltrating antigens in the CNS.

\section{Role of the blood-brain barrier in trafficking of immune cells}

The perceived inability of bone marrow derived cells to access the brain during steady-state conditions lead to the notion that the brain was sequestered from the immune system by the BBB. However, more recent studies have demonstrated that various immune cells in the CNS, most notably, activated monocytes, lymphocytes and B cells, can cross the BBB (Andersson et al, 1992; Nottet et al, 1996; Hickey et al, 1991; Knopf et al, 1998). Such studies have necessitated a re-thinking of the role of the BBB in immune cell trafficking into the central nervous system.
Monocyte and neutrophil trafficking across the $B B B$ An important part of the body's defense of bacterial or viral infections is the ability of monocytes and neutrophils to invade the tissue and begin phagocytosis of the foreign material. Indeed, infiltration of the monocytes and neutrophils from the bloodstream into the infected tissue is part of the initial immune response. However, there are notable differences in the penetration of monocytes and neutrophils in the BBB and the peripheral microvasculature. First, trafficking of activated leukocytes across the BBB display differences that are dependent on cell type, with monocytes being more capable than neutrophils in moving through the BBB. This was demonstrated in mice injected with various pro-inflammatory stimuli directly into the hippocampus (Andersson et al, 1991, 1992). Under normal homeostatic conditions, there was minimal margination and diapedisis of neutrophils and monocytes observed in the BBB. Following hippocampal injection of kianic acid or LPS challenge, a dramatic increase in the margination of neutrophils and monocytes to the BMVEC was seen (Andersson et al, 1991, 1992). Although there was an increased attachment of both neutrophils and monocytes to the BMVEC, only the monocytes underwent diapedisis and were able to move through the BBB into the brain. Infiltration of neutrophils through the BBB was only observed following breakdown of BBB integrity, and was in contrast to the rapid and substantial accumulation of neutrophils observed in the CSF through the choriod plexus (Andersson et al, 1991, 1992). The relative inability of neutrophils to move across the BBB, suggests that it is the initial monocyte response that is an important mediator of the immune response in the CNS.

Additional evidence in support of monocyte movement across the BBB can be found in the studies of Persidsky and colleagues (1997) and Fiala and co-workers (1997) examing the passage of the normal and stimulated monocytes across an in vitro model of the BBB. In these studies, the migration of HIV-infected monocytes was examined in a BBB model consisting of co-cultured BMVEC and astrocytes. As was observed in vivo, few monocytes were able to migrate across the BMVEC monolayer under normal conditions (Persidsky et al, 1997; Fiala et al, 1997). However, following activation of the monocytes with pro-inflammatory mediators such as LPS or tumor necrosis factor, significant increases in monocyte migration across the brain microvessel endothelial monolayers was observed (increases of up to 20-fold over control monolayers). Interestingly, there was no difference between HIV-infected or control (uninfected) monocyte migration across this BBB model, suggesting it is the generalized activation state of the monocyte that is important for penetrating the $\mathrm{BBB}$. 
Evidence for selectivity of migration of monocytes versus neutrophils in the in vitro BMVEC culture model has also recently been observed (Gan et al, 1999). In these studies the adhesion and migration of neutrophils and monocytes were examined in human BMVEC co-cultured with astrocytes. Following exposure of the monolayers to cocaine, there was an increase in adherence of both neutrophils and monocytes to the brain microvessel endothelial cells. Consistent with the in vivo studies, while both neutrophils and monocytes attached to the brain endothelial cells, only the monocytes exhibited significant migration across the endothelial cell monolayers (Gan et al, 1999).

These studies are in contrast to the early reports demonstrating significant adherence and migration of neutrophils across cultured bovine BMVEC monolayers (Dorovini-Zis et al, 1992). However, it should be noted that neutrophil migration was significantly increased in these studies through the use of specific chemotactic gradients produced by formyl-methionyl-leucyl phenylalanine, leukotriene B4 or acetyl-glyceryl-ether-phosphorylcholine. Such studies suggest that the activity of the monocyte migration observed in vivo may not be a function of the brain endothelial cells, but rather, the chemotactic agents presented in or around the CNS.

The time course required for the infiltration of monocytes and neutrophils into the CNS is also different from peripheral tissues. Following an inflammatory response outside the brain, there is a rapid infiltration of neutrophils and monocytes that occurs within minutes of the inflammatory stimulus. Peak migration rates for neutrophils and monocytes outside the brain typically occur within $4 \mathrm{~h}$ (Cybulsky et al, 1988). This is in stark contrast to the time course of infiltration observed in the CNS following acute inflammatory responses. Following direct injection of LPS into the parenchyma of the central nervous system of mice, Andersson and colleagues (1992) observed a delayed appearance of macrophages in the brain. In these studies there was no significant migration of monocytes into the brain parenchyma until 2 days post LPS injection in the hippocampus. While there was a significant increase in the number of macrophages in the brain 2 days after LPS stimulus, the number of macrophages were significantly less than observed in non-CNS tissue following LPS challenge (Andersson et al, 1992). The observation that the number of macrophage cells in the brain (following LPS injection) was reduced when peripheral circulating monocytes were depleted, suggests that a significant number of the macrophages in the brain parenchyma were attributable to the infiltration of monocytes across the BBB (Andersson et al, 1992). These studies indicate that the time course for entry of the monocytes into the brain is significantly delayed compared to other tissues outside the BBB.

Lymphocyte trafficking across the $B B B$

Under normal conditions, there are minimal numbers of lymphocytes detected within the CNS (Hauser et al., 1983; Hickey et al, 1991; Hickey and Kimura, 1987). This is the result of reduced penetration of lymphocytes across the BMVEC and the low levels of major histocompatibility complex expression in the normal CNS (Wong et al, 1984). However, during inflammatory conditions such as viral, bacterial or parasitic infections in the CNS, there is a significant accumulation of lymphocytes in the brain.

The ability of T lymphocytes to penetrate the BBB is illustrated in the studies of Hickey and colleagues (1991). In these studies, the penetration of $\mathrm{T}$ lymphocytes were examined in rat brain. The findings of these studies were that non-activated $\mathrm{T}$ lymphocytes had a minimal penetration into the CNS. However, activated $\mathrm{T}$ lymphocytes (i.e. lymphoblasts) were quite capable of penetrating the BBB (Hickey et al, 1991). The activated $\mathrm{T}$ lymphocytes crossed the BBB in a random fashion, and were not influenced by the specific antigen, major histocompatibility compatability, or phenotype of the T cell. Passage into the CNS occurred over a period of hours and, unless the specific antigen was recognized, the $\mathrm{T}$ lymphocytes exited the CNS within 24-48 h (Hickey et al, 1991). These studies suggest that activated immune cells can readily penetrate the $\mathrm{BBB}$. However, without recognition of specific antigen targets within the brain, the immune cells are recycled back to the blood.

There have been several in vitro studies demonstrating the penetration of $\mathrm{T}$ lymphocytes across BMVEC (Lou et al, 1996; Joseph et al, 1997; Adamson et al, 1999; Wong et al, 1999). In the studies of Wong and colleagues the penetration of lymphocytes was shown to occur both through movement across the cytoplasm of the BMVEC and through the tight junctions between the BMVEC. The lymphocyte penetration occurred without any apparent disruption of the integrity or solute permeability of the BMVEC monolayer (Wong et $a l, 1999)$. While the actual mechanism of penetration of immune cells across the BBB is discussed below, it should be noted that the in vitro studies are in agreement with the in vivo observations regarding lymphocyte penetration into the brain in that adherence and penetration is dependent on the generalized activation state of the immune cell and the BMVEC.

Less is known about the migration of B cells into the CNS. Evidence that B cells do enter the brain can be found in the studies of Hatalski et al (1998a,b) and Knopf et al (1998). In the studies by Hatalski 
and colleagues (1998a,b) the role of B cells in the humoral response to Borna disease was examined. The rats infected with Borna disease showed an acute immune response characterized by the migration of $\mathrm{T}$ cells and macrophages into the sites of infection in the CNS. There was also a chronic immune response which was observed in the rats infected with Borna disease. In the chronic stage, there was, among other things, a migration of B cells into the brain parenchyma (Hatalski et al, 1998a). The purpose of the B cell migration in the Borna virus infected rat was proposed to be the production of antibody in the CNS, as the BBB was intact and would not allow peripherally circulating antibodies to enter into the brain (Hatalski et al, 1998b).

Similar observations have recently been made regarding the movement of activated $\mathrm{B}$ cells into the brain by Knopf and co-workers (1998). In these studies, antigen was injected into the caudate nucleus of naive rats and rats pre-immunized against the antigen. Around the site of the cannula where the antigen was injected into the brain parenchyma of naive rats, the authors noted the presence of a scattering of B cells, as well as other leukocyte and lymphocyte cells (Knopf et al, 1998). However, in the pre-immunized rats, there was a much greater accumulation of antigen specific B cells, macrophages and $\mathrm{T}$ cells at the injection site within the caudate nucleus. In addition to the immune cells detected at the injection site, antibodies were also present within the CNS of the rats. Since there was no evidence of BBB disruption, the authors concluded that the antibodies detected in the CNS were the result of the movement of B cells into the brain (Knopf et al, 1998).

Importance of adhesion molecules in immune cell trafficking across the $B B B$

Identifying the cellular mechanisms by which the BBB allows the movement of the immune cells into the CNS is critical for understanding the immunology of the BBB. In this regard, attention has focused on the adhesion molecules found in the vascular endothelial cells that form the BBB. Some of these adhesion molecules, like the cadherins are involved in the formation of intercellular tight junction complexes between the brain microvessel endothelial cells. Other adhesion molecules form the selectins that are involved in the initial binding of the leukocytes to the endothelial cells. And still others in the integrin subclass have a role in the secondary adhesion process and subsequent migration of the various leukocytes across the endothelial cells. Of the three general classes of adhesion molecules, it is alterations in the selectin and integrin classes of adhesion molecules that most affect penetration of leukocytes across the BBB.

Early in the study of the cell adhesion molecules, it was suspected that the apparent selectivity with regard to leukocyte penetration of the BBB was due to the expression of BBB-specific cell adhesion molecules. However, more likely, the selective permeability of the BBB to the leukocytes is related, in part, to the level of expression of the various adhesion molecules during normal and inflammatory conditions. Immunohistochemical studies of frozen brain tissue show minimal to non-detectable levels of expression of various integrin adhesion molecules, including ICAM-1, VCAM-1 and PECAM-1 in the BBB (Williams et al, 1996; Washington et al, 1994; Engelhardt et al, 1994; Rossler et al, 1992). However, during activation of immune responses in the CNS, as seen in such conditions as MS or EAE, there is a significant up-regulation of the expression of ICAM-1, VCAM-1 and PECAM-1 in the brain microvessels (Williams et al, 1996; Washington et al, 1994; Engelhardt et al, 1994; Bo et al, 1996).

Studies with cultured BMVEC also indicate low levels of expression of ICAM-1 and VCAM-1 under normal unstimulated conditions (Lou et al, 1996; Nottet et al, 1996; Gan et al, 1999). Furthermore, there was a significant up-regulation of ICAM-1 and VCAM-1 expression in the cultured brain microvessel endothelial cells following exposure to proinflammatory agents or activated T cells (Lou et al, 1996; Nottet et al, 1996; Gan et al, 1999). These studies suggest that the increased penetration of monocytes and $\mathrm{T}$ cells into the central nervous system during activated or inflammatory conditions may be the result of increased expression of integrin adhesion molecules.

Alterations in the expression of the selectin group of adhesion molecules in the BBB during normal and inflammatory conditions is less conclusive. Studies by Egelhardt and colleagues (1997) in mice under both normal conditions and following the induction of EAE failed to detect the expression of mRNA for either E- or P-selectin in the brain microvessel endothelial cells. Furthermore, treatment of the mice with anti E- or P-selectin antibodies had no appreciable effect on the migration of activated T cells across the BBB (Engelhardt et al, 1997). This suggested that E- and P-selectin were neither constitutively expressed under normal conditions in the BBB or induced during inflammatory conditions in which the BBB becomes permeable to the immune cells.

In contrast, E-selectin has been detected in cultured human brain microvessel endothelia cells (Lou et al, 1996; Nottet et al, 1996; Wong et al, 1999). Furthermore, exposure of human brain microvessel endothelial cells to activated lymphocytes resulted in an increased expression of Eselectin (Lou et al, 1996). Evidence for the potential involvement of selectin adhesion molecules in the penetration of lymphocytes in the $\mathrm{BBB}$ is the observation that treatment of stimulated human BMVEC monolayers with E-selectin antibodies significantly reduced lymphocyte migration across 
the monolayer (Wong et al, 1999). In a different in vitro model, the adherence of monocytes to human brain tissue was reduced following treatment with E-selectin antibodies (Nottet et al, 1996). Combined treatment with both E-selectin and VCAM-1 antibodies reduced monocyte adherence to brain microvessels by greater than $70 \%$ indicating the potential involvement of both the selectin and integrin adhesion molecules in the adherence and translocation of monocytes across the BBB (Nottet et al, 1996).

In vitro studies using cultured human BMVEC also demonstrate reduced lymphocyte penetration following treatment with antibodies to ICAM-1, PECAM-1 or E-selectin (Wong et al, 1999). These studies highlighted the importance of an activated or 'primed' endothelial cell preparation, as lymphocyte adherence and penetration across unactivated human BMVEC monolayers was minimal. Interestingly, while adherence of $\mathrm{T}$ cells to TNFactivated BMVEC monolayers was dependent in part to VCAM-1, antibodies to VCAM-1 did not effect the migration of the lymphocytes across the monolayers (Wong et al, 1999).

Perhaps the most convincing evidence for the selectins involvement in the translocation of the immune cells across the BBB are the studies in selectin knock-out mice (Tang et al, 1996). In these studies, mice genetically deficient in P-selectin, or both $\mathrm{P}$ - and E-selectin were examined for their susceptibility to acute cytokine-induced meningitis. In these studies, the control mice treated with the cytokine developed severe meningeal inflammation that was characterized by significant increases in leukocyte accumulation in the central nervous system and enhanced BBB permeability (Tang et al, 1996). However, in the P-selectin knockout mice, leukocyte infiltration in the CNS and BBB permeability were significantly inhibited. Furthermore, in the doubly deficient P- and E-selectin mice, there was a nearly complete inhibition of leukocyte migration and BBB permeability (Tang et al, 1996). These studies suggest that the selectins do have a role in the increased penetration of immune cells in the CNS observed following inflammatory events in the brain.

While it is apparent that adhesion molecules have a major role in the margination and diapedesis of immune cells across the BMVEC, little is know about the second messenger systems that may be activated to facilitate the penetration of the immune cells across the BBB. In this regard, the studies of Adamson et al (1999) have shed light on cellular events which occur as a result of binding of lymphocytes to ICAM expressed in the BMVEC. By cross-linking the ICAM-1 expressed on an immortalized rat brain endothelial cell line with IgG directed at anti-ICAM-1 antibodies, Adamson and colleagues were able to demonstrate a clustering of ICAM-1 and an increase in actin stress fiber formation in the BMVEC. The stress fiber formation was associated with the activation of the small guanosine triphosphate-binding protein, Rho, suggesting that the Rho signalling pathway was involved in ICAM-1 mediated lymphocyte migration in the BBB. Further evidence for activation of Rho in the BMVEC for penetration of the immune cells is the experiments performed in the presence of the Rho inhibitor, C3 transferase. While activated T lymphocytes were still able to adhere to the BMVEC monolayers, treatment with C3 transferase significantly reduced the migration of the lymphocytes across the monolayer, the formation actin stress fibers, and the accumulation of Rho in the cells (Adamson et al, 1999). These studies indicate that the adhesion molecules, such as ICAM-1, not only bind to the lymphocytes as they travel through the bloodstream, but also activate the endothelial cell through various signalling pathways to facilitate migration of the lymphocytes across the BBB.

\section{Role of the blood-brain barrier as an immune response modifier}

It is clear that the brain endothelial cells are affected by a number of different pro-inflammatory mediators such as cytokines, prostaglandins, and bradykinin. In many cases, these pro-inflammatory agents have an important role in initiating the changes in the permeability or adhesion properties of the endothelial cells that allow the immune cells to infiltrate the CNS. However, in addition to their effects on BBB permeability, activation of the brain microvessel endothelial cells by various pro-inflammatory agents may serve other purposes important to the immune response.

Evidence for the BBB to act as an immune response modifier is illustrated in the studies of Quan et al (1997) and Herkenham et al (1998) in which the temporal expression of intracellular markers of cellular activation was examined following peripheral administration of either lipopolysaccharide or interleukin-1, respectively. In both these studies, there was a time dependent response to the pro-inflammatory agents, with increased expression of c-fos and inhibitory factor kappa $\beta$ alpha first observed (within 30-60 min) in the barrier cells of the choriod plexus and blood-brain barrier, and circumventricular organs that are not protected by the BBB (Quan et al, 1997; Herkenham et al, 1998). Interestingly, following the initial burst in activity observed in the brain microvasculature, the microvessel endothelial cells returned to their quiescent state, and a delayed increase in activity was observed in the cells within the CNS that were adjacent to the BBB. The temporal cellular activation pattern observed, prompted the authors to suggest that peripheral immune response modifiers 
may activate cells within the CNS by secondary mediators released from the cells of the BBB and blood-cerebral spinal fluid barrier.

In considering the identify of the secondary immune response modulators released from the brain microvessel endothelial cells there are a multitude of potential candidates. The vascular endothelium expresses several different cytokine and cytokine receptors. The demonstration that exposure of human brain microvessel endothelial cell monolayers to stimulated $\mathrm{T}$ lymphocytes results in the secretion of interleukin 6 and interleukin 8 from the endothelial cells supports the contention that cytokines released from the BBB may act as secondary immune response modulators (Lou et al, 1996). The cytokines released from the brain endothelial cells may act to modulate activity of themselves or other nearby cells such as the astrocytes and glial cells. Other potential immune modulators released by the endothelial cells of the BBB are arachidonic acid metabolites such as prostaglandins and thromboxanes. Support for the possible involvement of the prostaglandins is the studies by Quan and coworkers (1998) that demonstrated an induction in cyclooxygenase 2 , one of the isoenzymes involved in prostaglandin production, in brain microvessel endothelial cells several hours after exposure to bacterial LPS or cytokines. A similar induction of cyclooxygenase 2 with a resulting increased release of prostaglandin $\mathrm{E}$, has been observed in our laboratory in primary cultured bovine brain microvessel endothelial cell monolayers following treatment with tumor necrosis factor (manuscript in preparation).

The concept of the BBB acting as a relay point for either amplifying or dampening immune responses in the brain is an area that is underdeveloped. Most attention has focused on the role of the BBB in allowing the penetration of immune cells into the CNS. While immune cell trafficking across the BBB is an important event, the BMVEC that form the BBB also have the ability, through increased permeability, altered release, or cell-to-cell junctional communication, to control the immuno-responsiveness of the brain.

\section{Summary}

The BMVEC forming the BBB have an important role in mediating immune responses in the central nervous system. Given the anatomical location of the brain microvessel endothelial cells, they represent a point of passage for immune cells from the systemic blood into the extracellular environment of the brain. While normal trafficking of the immune cells across the BBB is limited, under the appropriate conditions, the brain microvessel endothelial cells can allow the selective entry of leukocytes into the central nervous system. Characterization of the stimuli required to produce the change in BBB permeability to the cells of the immune system and the cellular factors involved in such changes are areas of intense examination. Both in vivo and in vitro studies suggest the increased passage of immune cells across the BBB during inflammatory conditions involve changes in the expression of adhesion molecules in the endothelial cells that form the BBB. The presence of various chemokines may also contribute to the permeability of the BBB to select leukocytes observed during inflammatory conditions.

The brain microvessel endothelial cells also act as an immune response modifier, releasing agents that in turn act upon cells within the central nervous system. One important target of these agents released from the brain microvessel endothelial cells is the microglia. The importance of this can not be overlooked as modification of the activity of the microglial cells, the resident monocytes of the central nervous system, would be expected to play an important role in immune responses in the brain. In this regard, the release of endogenous immune response modifiers (i.e. cytokines, prostaglandins, etc) by the endothelial cells in the BBB may act to either intensify or inhibit the propagation of the immune response in the brain.

\section{Acknowledgements}

Financial support provided to the author by USPHS grant R29 NS-36831. The author wishes to thank Dr Howard Gendelman for the helpful comments in the preparation of the manuscript.

\section{References}

Adamson P, Etienne S, Couraud P-O, Calder V, Greenwood J (1999). Lymphocyte migration through brain endothelial cell monolayers involves signaling through endothelial ICAM-1 via a rho-dependent pathway. J Immunol 162: 2964-2973.

Andersson PB, Perry VH, Gordon S (1991). The kinetics and morphological characteristics of the macrophagemicroglial response to kainic acid-induced neuronal degeneration. Neuroscience 42: 201-214. 
Andersson PB, Perry VH, Gordon S (1992). The acute inflammatory response to lipopolysaccharide in CNS parenchyma differs from that in other body tissues. Neuroscience 48: 169-186.

Angelov DN, Walther M, Streppel M, Guntinas-Lichius O, Neiss WF (1998). The cerebral perivascular cells. Adv Anat Embryol Cell Biol 147: 1-87.

Audus KL, Chikhale PJ, Miller DW, Thompson SE, Borchardt RT (1992). Brain uptake of drugs: The influence of chemical and biological factors. Adv Drug Res 23: 3-64.

Bartus RT, Elliott PJ, Dean RL, Hayward NJ, Nagle TL, Huff MR, Snodgrass PA, Blunt DG (1996). Controlled modulation of $\mathrm{BBB}$ permeability using the bradykinin agonist, RMP-7. Exp Neurol 142: 14-28.

Bo L, Peterson JW, Mork S, Hoffman PA, Gallatin WM, Ransohoff RM, Trapp BD (1996). Distribution of immunoglobulin superfamily members ICAM-1, -2, -3 , and the beta 2 integrin LFA-1 in multiple sclerosis lesions. J Neuropathol Exp Neurol 55: 1060-1072.

Brightman MW (1989). The anatomic basis of the bloodbrain barrier. In: Implications of the Blood-Brain Barrier and Its Manipulation, Vol. 1. Neuwelt E (ed). Plenum Press: New York, pp 53-83.

Broadwell RD (1989). Trancytosis of macromolecules through the blood-brain barrier: A cell biological perspective and critical appraisal. Acta Neuropathol 79: $117-128$.

Cserr HF, Knopf PM (1992). Cervical lymphatics, the blood-brain barrier and the immunoreactivity of the brain: a new view. Immunol Today 13: 507-512.

Cybulsky MI, McComb DJ, Movat HZ (1988). Neutrophil leukocyte emigration induced by endotoxin. I Immunol 140: $3144-3149$.

Czech KA, Ryan JW, Sagen J, Pappas GD (1997). The influence of xenotransplant immunogenicity and immunosuppression on host MHC expression in the rat CNS. Exp Neurol 147: 66-83.

Dorovini-Zis K, Bowman PD, Prameya R (1992). Adhesion and migration of human polymorphonuclear leukocytes across cultured bovine brain microvessel endothelial cells. J Neuropathol Exp Neurol 51: 194205.

Engelhardt B, Conley FK, Butcher EC (1994). Cell adhesion molecules on vessels during inflammation in the mouse central nervous system. J Neuroimmunol 51: $199-208$

Engelhardt B, Vestweber D, Hallmann R, Schulz M (1997). E- and P-selectin are not involved in the recruitment of inflammatory cells across the bloodbrain barrier in experimental autoimmune encephalomyelitis. Blood 90: 4459-4472.

Epstein LG (1998). HIV neuropathogenesis and therapeutic strategies. Acta Paediatr Jpn 40: 107-111.

Fabry Z, Fitzsimmons KM, Herlien JA, Moninger TO, Dobbs MB, Hart MN (1993). Production of the cytokines interleukin 1 and 6 by murine brain microvessel endothelium and smooth muscle pericytes. $J$ Neuroimmunol 47: 23-34.

Fiala M, Looney D, Stins M, Way DD, Zhang L, Gan X, Chiappelli F, Schweitzer ES, Shapshak P, Weinand M, Graves MC, Witte M, Kim KS (1997). TNF-alpha opens a paracellular route for HIV-1 invasion across the blood-brain barrier. Mol Med 3: 553-564.
Gan X, Zhang L, Berger O, Stins MF, Way D, Taub DD, Chang SL, Kim KS, House SD, Weinand M, Witte M, Graves MC, Fiala M (1999). Cocaine enhances brain endothelial adhesion molecules and leukocyte migration. Clin Immunol 91: 68-76.

Goldstein GW (1988). Endothelial cell-astrocyte interactions. Ann NY Acad Sci 529: 31-39.

Greenwood J, Adu J, Davey AJ, Abbott NJ, Bradbury MW (1991). The effect of bile salts on the permeability and ultrastructure of the perfused, energy-depleted, rat blood-brain barrier. J Cereb Blood Flow Metab 11: $644-654$.

Greig NH (1989). Drug delivery to the brain by bloodbrain circumvention and drug modification. In: Implications of the Blood-Brain Barrier and Its Manipulation, Vol. 1. Neuwelt E (ed). Plenum Press: New York, pp 311-368.

Hatalski CG, Hickey WF, Lipkin WI (1998a). Evolution of the immune response in the central nervous system following infection with Borna disease virus. $J$ Neuroimmunol 90: 137-142.

Hatalski CG, Hickey WF, Lipkin WI (1998b). Humoral immunity in the central nervous system of Lewis rats infected with Borna disease virus. J Neuroimmunol 90: $128-136$.

Hauser SL, Bhan AK, Gilles GH, Hoban CJ, Reinherz EL, Weiner HL (1983). Immunohistochemical staining of human brain with monoclonal antibodies that identify lymphocytes, monocytes and the Ia antigen. I Neuroimmunol 5: $197-205$.

Hayashi Y, Nomura M, Yamagishi S, Harada S, Yamashita J, Yamamoto $H$ (1997). Induction of various blood-brain barrier properties in non-neural endothelial cells by close apposition to co-cultured astrocytes. Glia 19: $13-26$.

Herkenham M, Lee HY, Baker RA (1998). Temporal and spatial patterns of c-fos mRNA induced by intravenous interleukin-1: A cascade of non-neuronal cellular activation at the blood-brain barrier. J Comp Neurol 400: $175-196$.

Hickey WF (1999). Leukocyte traffic in the central nervous system: the participants and their roles. Semin Immunol 11: 125-137.

Hickey WF, Hsu BL, Kimura H (1991). T-lymphocyte entry into the central nervous system. J Neurosci Res 28: $254-260$.

Hickey WF, Kimura H (1987). Graft-versus-host disease elicits expression of class I and class II histocompatibility antigens and the presence of scattered T-cells in rat central nervous system. Proc Natl Acad Sci USA 84: $2082-2087$.

Hurst RD, Clark JB (1998). Alterations in transendothelial electrical resistance by vasoactive agonists and cyclic AMP in a blood-brain barrier model system. Neurochem Res 23: 149-154.

Hurwitz AA, Berman JW, Rashbaum WK. Lyman WD (1993). Human fetal astrocytes induce the expression of blood-brain barrier specific proteins by autologous endothelial cells. Brain Res 625: 238-243.

Joseph J, Lublin FD, Knobler RL (1997). Modulation of T cell-endothelial adhesion by astrocyte conditioned medium. Glia 21: 408-412. 
Knopf PM, Harling-Berg CJ, Cserr HF, Basu D, Sirulnick EJ, Nolan SC, Park JT, Keir G, Thompson EJ, Hickey WF (1998). Antigen-dependent intrathecal antibody synthesis in the normal rat brain: Tissue entry and local retention of antigen-specific B cells. J Immunol 161: $692-701$

Lou J, Dayer JM, Grau GE, Burger D (1996). Direct cell/ cell contact with stimulated $\mathrm{T}$ lymphocytes induces the expression of cell adhesion molecules and cytokines by human brain microvascular endothelial cells. Eur J Immunol 26: 3107-3113.

Mark KS, Miller DW (1999). Increased permeability of primary cultured brain microvessel endothelial cell monolayers following TNF- $\alpha$ exposure. Life Sci 64: $1941-1953$.

Nottet HSLM, Persidsky Y, Sasseville VG, Nukuna AN, Bock P, Zhai Q-H, Sharer LR, Mccomb RD, Swindells S, Soderland C, Gendelman HE (1996). Mechanisms for the transendothelial migration of HIV-1-infected monocytes into brain. J Immunol 156: 1284-1295.

O’Keffe GM, Nguyen VT, Benveniste EN (1999). Class II transactivator and class II MHC gene expression in microglia: modulation by the cytokines TGF-beta, IL-4, IL-13 and IL-10. Eur J Immunol 29: 1275-1285.

Persidsky Y, Stins M, Way D, Witte MH, Weinand M, Kim KS, Bock P, Gendelman HE, Fiala M (1997). A model for monocyte migration through the blood-brain barrier during HIV-1 encephalitis. J Immunol 158: $3499-3510$.

Power C, Johnson RT (1995). HIV-1 associated dementia: clinical features and pathogenesis. Can J Neurol Sci 22: $92-100$.

Quan N, Whiteside M, Herkenhan M (1998). Cyclooxygenase 2 mRNA expression in rat brain after peripheral injection of lipopolysaccharide. Brain Res 802: $189-197$.

Quan N, Whiteside M, Kim L, Herkenham M (1997). Induction of inhibitory factor $\kappa \mathrm{B} \alpha$ mRNA in the central nervous system after peripheral lipopolysaccharide administration: An in situ hybridization histochemistry study in the rat. Proc Natl Acad Sci USA 94: 10985-10990.

Ransohoff RM, Tani M (1998). Do chemokines mediate leukocyte recruitment in post-traumatic CNS inflammation? Trends Neurosci 21: 154-159.

Raub TJ, Kuentzel SL, Sawada GA (1992). Permeability of bovine brain microvessel endothelial cells in vitro: Barrier tightening by a factor released from astroglioma cells. Exp Cell Res 199: $330-340$.

Rist RJ, Romero IA, Chan MW, Couraud PO, Roux F, Abbott NJ (1997). F-actin cytoskeleton and sucrose permeability of immortalised rat brain microvascular endothelial cell monolayers: effects of cyclic AMP and astrocytic factors. Brain Res 768: 10-18.
Rossler K, Neuchrist C, Kitz K, Scheiner O, Kraft D, Lassman H (1992). Expression of leucocyte adhesion molecules at the human blood-brain barrier (BBB). J Neurosci Res 31: 365-374.

Rubin LL, Hall DE, Porter S, Barbu K, Cannon C, Horner HC, Janatpour M, Liaw CW, Manning K, Morales J, Tanner LI, Tomaselli KJ, Bard FJ (1991). A cell culture model of the blood-brain barrier. J Cell Biol 115: $1725-1735$.

Schneeberger EE, Lynch RT (1984). Tight junctions, their structure, composition and function. Circ Res 55: $723-733$

Sharief MK, Noori MA, Ciardi M, Cirelli A, Thompson EJ (1993). Increased levels of circulating ICAM-1 in serum and cerebrospinal fluid of patients with active multiple sclerosis. Correlation with TNF-a and bloodbrain barrier damage. J Neuroimmunol 43: 15-22.

Spellerberg B, Tuomanen EI (1994). The pathophysiology of pneumococcal meningitis. Ann Med 26: 411-418.

Stewart PA, Wiley MJ (1985). Developing nervous tissue induces formation of blood-brain barrier characteristics in invading endothelial cells: A study using quailchick transplantation chimeras. Dev Biol 84: 184-192.

Tang T, Frenette PS, Hynes RO, Wagner DD, Mayadas TN (1996). Cytokine-induced meningitis is dramatically attenuated in mice deficient in endothelial selectins. J Clin Invest 97: 2485-2490.

Washington R, Burton J, Todd RF, Newman W, Dragovic L, Dore-Duffy P (1994). Expression of immunologically relevant endothelial cell activation antigens on isolated central nervous system microvessels from patients with multiple sclerosis. Annals Neurol 35: $89-97$.

Williams KC, Zhao RW, Ueno K, Hickey WF (1996). PECAM-1 (CD31) expression in the central nervous system and its role in experimental allergic encephalomyelitis in the rat. J Neurosci Res 45: 747-757.

Wong D, Prameya R, Dorovini-Zis K (1999). In vitro adhesion and migration of $\mathrm{T}$ lymphocytes across monolayers of human brain microvessel endothelial cells: regulation by ICAM-1, VCAM-1, E-selectin and PECAM-1. J Neuropathol Exp Neurol 58: 138-152.

Wong GH, Bartlett PF, Clark-Lewis I, Battye F, Schrader JW (1984). Inducible expression of $\mathrm{H}-2$ and Ia antigens on brain cells. Nature 310: 688-691. 